\title{
Ordered Two-Dimensional Superstructures of Colloidal Octapod- Shaped Nanocrystals on Flat Substrates
}

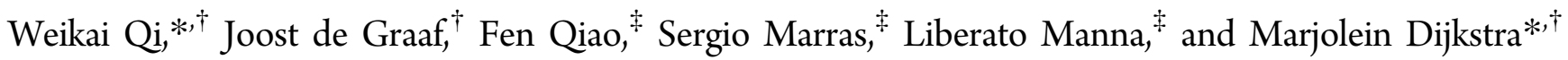 \\ ${ }^{\dagger}$ Soft Condensed Matter, Debye Institute for Nanomaterials Science, Utrecht University, Princetonplein 5, 3584 CC Utrecht, The \\ Netherlands \\ ${ }^{\ddagger}$ Istituto Italiano di Tecnologia (IIT), Via Morego 30, 16163 Genova, Italy
}

Supporting Information

\begin{abstract}
We studied crystal structures in a monolayer consisting of anisotropic branched colloidal (nano)octapods. Experimentally, octapods were observed to form a monolayer on a substrate with a square-lattice crystal structure by dropcasting and fast evaporation of solvent. The experimental results were analyzed by Monte Carlo simulations using a hard octapod model consisting of four interpenetrating spherocylinders. We confirmed by means of free-energy calculations that crystal structures with a (binary-lattice) square morphology are indeed thermodynamically stable at high densities. The effect of the pod

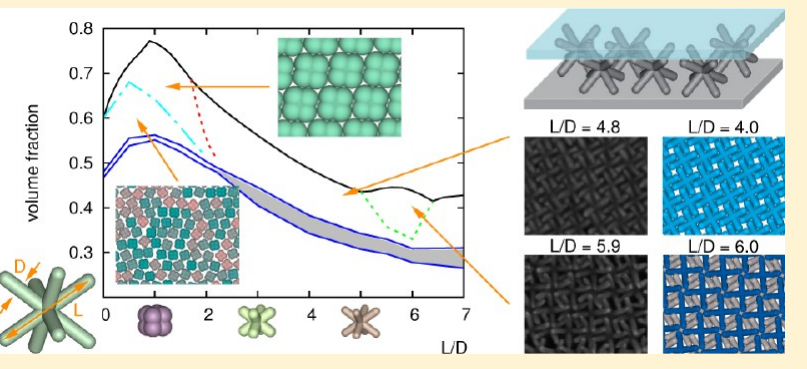
length-to-diameter ratio on the crystal structures was also considered and we used this to constructed the phase diagram for these hard octapods. In addition to the (binary-lattice) square crystal phase, a rhombic crystal and a hexagonal plastic-crystal (rotator) phase were obtained. Our phase diagram may prove instrumental in guiding future experimental studies.
\end{abstract}

KEYWORDS: Octapods, nanocrystals, quasi-2D, self-assembly, anisotropy, colloids

$\mathrm{T}$ he study of nanocrystal monolayers offers many opportunities for the creation of new materials with bulk properties that differ substantially from the materials that form by self-assembly in three dimensions. ${ }^{1,2}$ This has led to a strong experimental and simulation interest in the behavior of nanocrystals in a (quasi-)2D geometry, that is, three-dimensional (3D) particles confined to a two-dimensional (2D) geometry. For instance, the seemingly simple system consisting of monodisperse hard disks in a $2 \mathrm{D}$ plane has sparked intense debate on the nature of the $2 \mathrm{D}$ solid-liquid phase transition. $^{3-6}$ In addition, experiments and simulations ${ }^{7-14}$ showed that rod- and square-shaped convex anisotropic particles display a rich mesophase behavior when confined to a (quasi-)2D geometry.

Advances in the synthesis of colloids and nanocrystals have resulted in monodisperse samples consisting of complex particles with anisotropic hard and soft interactions ${ }^{15-21}$ and present many possibilities for further development in this field. Moreover, new simulation techniques are available to study the experimentally observed phenomenology for these new particles and to tackle the complex numerical problems such investigations bring about. ${ }^{22-31}$ Only recently has the investigation into the phase behavior of anisotropic particles in (quasi-)2D by simulation been extended to the realm of nonconvex particles. ${ }^{29}$ However, studying the phase behavior of nonconvex particles under confinement remains challenging due to geometric restrictions and the complex interactions between the particles. ${ }^{32}$
Our group recently reported an experimental and simulation study of the hierarchical self-assembly of anisotropic branched colloidal nanocrystals, so-called octapods, into 3D superstructures in the liquid bulk phase. ${ }^{20}$ In this Letter, we extend our findings to the formation of monolayers consisting of octapods, which were obtained by a deposition-evaporation procedure. In the experiments, we obtained monolayers in the micrometer size range in which the octapods with a pod lengthto-diameter ratio of $L / D \approx 4.8$ arranged into a square-lattice crystal (SC). Here, we view the octapod as four interpenetrating pods of length $L$ and diameter $D$. In addition, we observed the formation of a monolayer with pieces of a binarylattice square crystal (BSC) for $L / D \approx 5.9$. That is, a crystal with a square-lattice structure for which neighboring particles have a fixed orientational difference, resulting in two square sublattices with different particle orientations. We also studied the formation of these monolayers using Monte Carlo (MC) simulations, where we modeled the octapods using four interpenetrating hard spherocylinders, which were constrained to move in a quasi-2D geometry. We found the high density phase to be a SC or a BSC for values of $L / D$ comparable to the experiments. For lower $L / D$ we observed a high-density rhombic crystal (RC). Using free-energy calculations, we were able to establish the phase diagram for our hard octapod model. We observed a first-order phase transition between the SC and

Received: July 16, 2012

Revised: August 30, 2012

Published: August 31, 2012 
the isotropic liquid (IL) phase. We also found a continuous phase transition from the BSC to the SC in which only the particle orientations changed, but the crystal lattice remains unaffected. Finally, we found that the RC phase melted into the IL via a hexagonal plastic-crystal (rotator) phase (HR).

The octapod-shaped nanocrystals used in the experiments were synthesized according to literature procedures, ${ }^{19,20}$ also see the Supporting Information. They are made of a central core of CdSe and pods of CdS. These nanocrystals are coated with a monolayer of surfactants (mainly alkyl phosphonic acids), which make them soluble in nonpolar or moderately polar solvents. The assemblies were prepared by drop-casting a solution of octapods in toluene $\left(10^{-8} \mathrm{M}\right)$ on a substrate, after which the solvent was allowed to evaporate at room temperature. Evaporation of the solvent varied from sample to sample, but in general did not take more than a few minutes. The sample was then thermally annealed at $200{ }^{\circ} \mathrm{C}$. We tested the formation of the assemblies on various substrates.

When the samples were analyzed by scanning electron microscopy (SEM), we found that in general different regions of the substrate presented different types of octapod organization. First, chains of interlocked octapods were found all over the substrate where the solution had been deposited, even though they were predominant at the "coffee stain" region (Figure 1a). ${ }^{33}$ This represents a stripe of accumulated material,
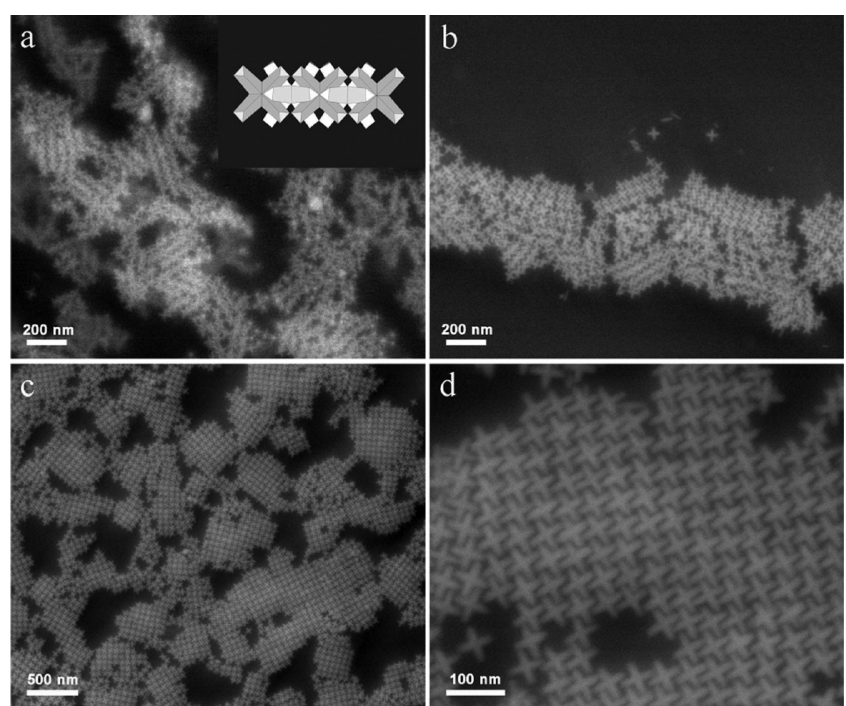

Figure 1. High-resolution SEM low-angle backscattered electron images of octapods with length-to-diameter ratio $(L / D)$ of 4.8 deposited on a $\mathrm{Si}_{3} \mathrm{~N}_{4}$ membrane. (a) Coffee stain region containing mostly chains of octapods with interlocked configuration (sketched in the inset) embedded in an organic matrix; (b) region with coexisting square-lattice crystals and packed chains; (c,d) different magnifications of the central area delimited by the coffee stain. This area is predominantly covered with square-lattice crystals. The scale bars in (a-d) are 200, 200, 500, and $100 \mathrm{~nm}$, respectively.

including nanocrystals and often also excess organics, that was generated during the evaporation of the solvent and that delimited the whole region of the substrate where the original droplet was sitting. It is very likely that the chains of interlocked octapods were already present in the solution prior to deposition, as shown by us in our previous work. ${ }^{20}$ A plausible explanation for their accumulation at the coffee stain region is that they were preferentially dragged, by convective flow, toward the contact line of the drying solution with the substrate. This is the region where the retracting droplet pins and depins during solvent evaporation and where the coffee stain of accumulated material is formed.

In general, no square-lattices (SCs) of octapods were found at the coffee stain region. Instead, they were frequent in the inner regions of the substrate delimited by the coffee stain. These are the locations on the substrate where material is deposited during the last stages of solvent evaporation, i.e., are the last locations from where the solution retracted prior to complete evaporation. Here we found areas in which SCs coexisted with chains (Figure $1 \mathrm{~b}$ ) and areas that were covered only with SCs (Figure 1c,d). It is therefore likely that at these stages the solution reaches a high concentration of octapods and they start self-assembling. This assembly presumably takes place with the constraint imposed by the substrate that restricts octapod motion and rotation only to one plane, because we observed that the octapods favorably touch the substrate with four pod tips. In this specific sample, the substrate was a $\mathrm{Si}_{3} \mathrm{~N}_{4}$ membrane, the pods of the octapod had an average $L / D$ of 4.8 , and the unit cell in the SCs had a lattice size of $40 \mathrm{~nm}$. Deposition on various other substrates gave similar results (see Figure 2 and also the Supporting Information). In general, the

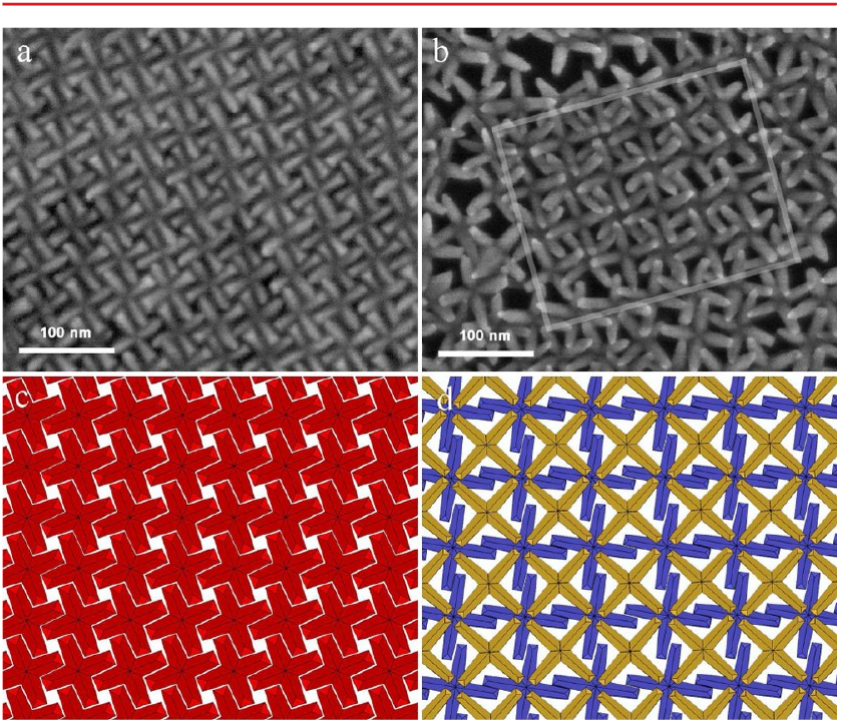

Figure 2. High-resolution SEM secondary electron images and related models showing the influence of the length-to-diameter ratio $(L / D)$ on the organization of the octapods. $(\mathrm{a}, \mathrm{c})$ For $L / D=4.8$, only simple square-lattice crystals were formed, while $(b, d)$ for $L / D=5.9$ binarylattice square crystals were occasionally found, as indicated by the outline in (b). The scale bars are $100 \mathrm{~nm}$.

octapods were organized into simple SCs, like the ones shown in Figure 1c,d and Figure 2a (in the latter case, the substrate is highly oriented pyrolytic graphite (HOPG) and the $L / D$ of the pods is again 4.8 ). However, for $L / D \approx 5.9$ we also found evidence of binary-lattice square crystals (BSCs), as predicted by our simulations; this is shown in Figure $2 b$.

To help understand the experimental findings, we performed MC simulations for which we modeled the octapod-shaped nanocrystals by four hard interpenetrating spherocylinders. These models were constrained to move in a quasi-2D geometry where the tips touch a flat substrate, see the Supporting Information for further details. The model is completely described by the length-to-diameter ratio $L / D$ of the spherocylinders with $L$ the length (excluding the hemi- 

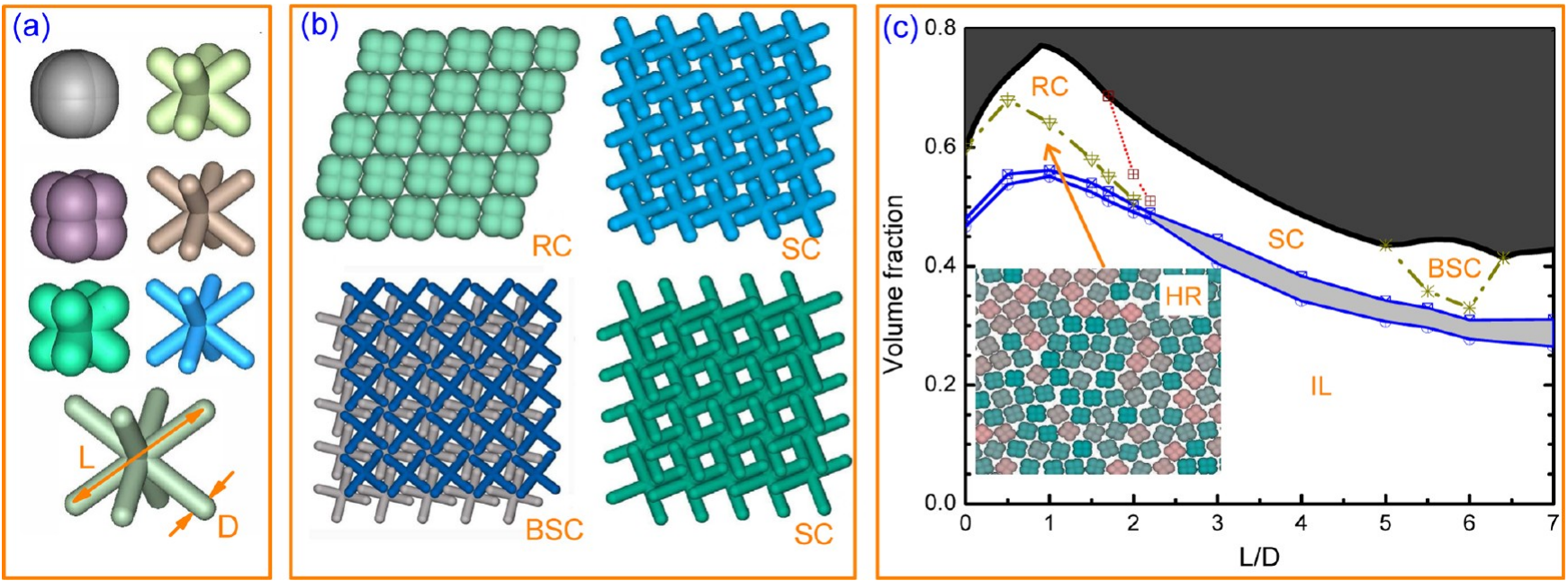

Figure 3. (a) The hard spherocylinder-based octapod model for different length-to-diameter ratios: $L / D=0.2,1.0$, and 2.0 (left column); and $L / D=$ 3.0, 5.0, and 6.0 (right column); the bottom octapod $(L / D=7.0$ ) shows our length $L$ and diameter $D$ definition. (b) Top views of quasi-2D densepacked structures obtained for various $L / D$ values. A rhombic crystal $(\mathrm{RC})$ for $L / D=1.0$, a noninterlocking square-lattice crystal $(\mathrm{SC})$ for $L / D=$ 4.0, a binary-lattice square crystal (BSC) for $L / D=6.0$, and an interlocking $S C$ for $L / D=7.0$ are shown. For the BSC, the two octapod orientations are accentuated using color. (c) The $L / D$-volume fraction phase diagram for hard octapods in a quasi- $2 \mathrm{D}$ system. The light-gray area indicates the coexistence region and the dark-gray area indicates the forbidden region above the maximum packing fraction (thick black line). We show the stable hexagonal plastic-crystal (rotator) phase (HR) in the inset; the color illustrates the octapods' orientation. The blue circles indicate the isotropic liquid (IL) phase-coexistence volume fraction, the blue squares the HR and SC coexistence volume fractions. The solid blue lines are a guide to the eye. The SC-BSC transition is indicated by brown stars and thin dash-dotted lines, The RC-HR transition by brown triangles and thick dash-dotted lines, and the RC-SC transition by red squares and red dashed lines.
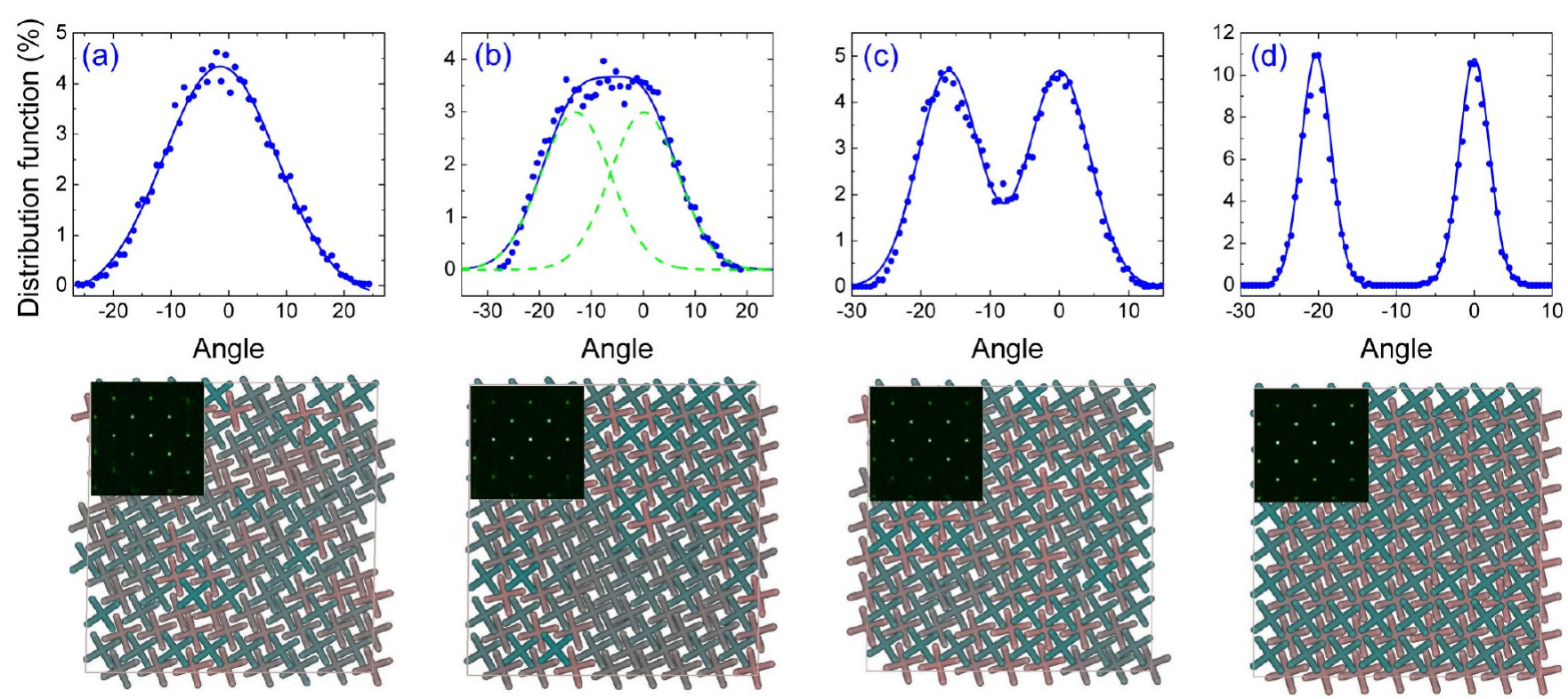

Figure 4. The distribution of the difference in orientation (in degrees) between neighboring octapods with $L / D=6.0$ for several values of the reduced pressure $P^{*}=P A / k_{\mathrm{B}} T$, where $k_{\mathrm{B}}$ is Boltzmann's constant, $T$ is the temperature, $P$ is the pressure, and $A$ is the planar area enclosed by the box. We also show snapshots and structure factors (insets) to illustrate the state of the system: (a) $P^{*}=0.230$, (b) $P^{*}=0.260,(\mathrm{c}) P^{*}=0.280$, and (d) $P^{*}=0.450$. The blue dots show measured values for the distribution; the blue lines show a single or double-Gaussian fit to the simulation results. The dashed green lines in (b) give the distribution function obtained by a double-Gaussian fit.

spherical caps) and $D$ the diameter. Figure 3a shows our model for several choices of $L / D$. Note that for $L / D=0$ the model reduces to a sphere. Using our model and a crystal-structure prediction algorithm similar to that of refs 28 and 34, we were able to show that the high-density structures observed in the experiments can be explained by excluded volume interactions only. Here we assumed that the van der Waals (vdW) interactions between octapods ${ }^{20}$ are dominated by the aggregation forces that occur during solvent evaporation, thereby allowing for an accurate description using a hard particle model. Figure $3 b$ shows a visual representation of the various high-density crystal structures that we obtained by varying $L / D \in[0,8]$. The corresponding (maximum) packing fraction is shown in the phase diagram of Figure $3 c$, where it is denoted by the boundary of the black region. We obtained the following high-density structures: rhombic crystals (RCs) for $L / D \in[0.0,1.7]$, square-lattice crystals (SCs) for $L / D \in$ $[1.8,5.0] \cup[6.3,8.0]$, and binary-lattice square crystals (BSCs) 
for $L / D \in[5.1,6.2]$. Note that for $L / D \gtrsim 6.3$ the SCs have an "interlocking" property (they appear to overlap in the top view, also see the Supporting Information).

By determining the equations of state (EOSs) for several conveniently chosen $L / D$ and performing free-energy calculations we were able to establish the full phase diagram of hard octapods in our quasi-2D system; see Figure 3c. In addition to the RC, SC, and BSC phase, we found an isotropic liquid (IL) and a hexagonal plastic crystal (rotator) phase (HR). For the SC-IL transition, we found a density jump and we confirmed the presence of a first order phase transition. We observed that the BSC melts into a SC, which subsequently melts into the IL phase upon lowering the pressure. The stability of the BSC at high pressures was further shown by preparing systems in a SC arrangement and allowing these to evolve: in all cases, the SC rearranged to form a BSC. The BSC-SC (solid-solid) phase transition appeared continuous; both the EOS and the free energy did not show a jump within the error bar.

Remarkably, during the BSC-SC transition only the orientation of the particles changed, while the lattice itself remained unaffected. We used this orientational dependence to identify the BSC-SC transition point by examining the distribution of the orientation difference between neighboring particles. Figure 4 shows four of these distributions at several pressures for octapods with $L / D=6.0$. In the SC (Figure 4a), we obtained a single-peak Gaussian distribution, because all particles have nearly the same orientation. For the BSC phase, the distribution has a double-peak Gaussian nature, because there are two different orientations, one for each (square) sublattice (Figure 4c,d). Near the BSC-SC phase transition, the two peaks merge into a single plateau (Figure $4 \mathrm{~b}$ ). We also calculated the structure factor based on the center-of-mass position of each particle, which showed that the square-lattice structure does not change during the SC-BSC transition.

The $\mathrm{RC}$ was found to be the stable phase at high pressures for octapods with $L / D \in[0.0,1.7]$, however the phase appeared to persist for $L / D \in[0.0,2.2]$ at lower pressures. For $L / D=$ 1.0 , the RC melted into a HR phase upon lowering the pressure. The EOS appeared continuous within our numerical precision. We therefore located the solid-solid transition point using the susceptibility of 4-fold bond orientational order parameter $\chi_{4}$, which showed divergent behavior at the phase transition. ${ }^{35}$ The HR phase melted into the IL phase upon further lowering the pressure. We demonstrated that this phase transition is first order. For octapods with $L / D=2.0$, the densest structure is an SC. This SC melts into a RC at lower pressures, which in turn melts into the $\mathrm{HR}$ and eventually into the IL phase. The SC-RC phase transition appeared to be continuous (possibly weak first order) within the numerical error.

In conclusion, we have shown that it is possible to prepare monolayers consisting of octapod-shaped nanocrystals arranged in a square-lattice crystal by drop-casting a suspension of octapods on a substrate and allowing the solvent to evaporate. The formation of square lattices is favored by the presence of the substrate, which constrains octapod movement and rotation to only one plane. Further improvements in the experimental conditions might allow us to achieve square lattices that are larger than those reported in the present work. These larger 2D assemblies might be of use in device applications. For instance, one monolayer of ordered octapods could be used to support a sheet of graphene to promote charge transfer from the sheet to the substrate, while keeping the substrate and graphene sheet separated. Another potential use is as a templating agent, by which the geometry of a second layer of particles can be manipulated. To confirm that the experimentally observed structures could indeed form at high pressures based on excluded-volume considerations only, we applied a variety of quasi-2D MC simulation techniques on a hard-particle model consisting of four interpenetrating hard spherocylinders. We extended upon our high-pressure simulation results by determining the full equilibrium phase diagram for octapods constrained to this particular quasi-2D geometry upon varying the length-to-diameter ratios for the constituent spherocylinders. In addition to the isotropic liquid phase, we found a variety of crystal phases: a rhombic crystal, a square-lattice crystal, a binary-lattice square crystal, and a hexagonal plasticcrystal (rotator) phase. Using free-energy calculations and by examining global bond-orientational order parameters, as well as the associated susceptibilities, we were able to establish the nature of the various solid-solid and solid-liquid phase transitions. Our results are useful for future experimental studies of monolayers consisting of anisotropic branched nanocrystals and are an initial step toward describing the (out-of-equilibrium) formation of the experimentally observed monolayers.

\section{ASSOCIATED CONTENT}

\section{Supporting Information}

Additional information and figures. This material is available free of charge via the Internet at http://pubs.acs.org.

\section{AUTHOR INFORMATION}

\section{Corresponding Author}

*E-mail: (W.Q.) W.Qi@uu.nl; (M.D.) M.Dijkstra1@uu.nl.

\section{Notes}

The authors declare no competing financial interest.

\section{ACKNOWLEDGMENTS}

It is a pleasure to thank Dr. G. Bertoni, Dr. R. Brescia, A. P. Gantapara, Dr. R. Ni, Dr. F. Smallenburg, Dr. D. Ashton, and Dr. R. van Roij for useful discussions. We would also like to thank K. Miszta for help with the synthesis of octapods. M.D. acknowledges financial support by a "Nederlandse Organisatie voor Wetenschappelijk Onderzoek" (NWO) Vici Grant, and J.dG. by the Utrecht University High Potential Programme. L.M. acknowledges financial support from the European Union through the FP7 starting ERC Grant NANO-ARCH (contract no. 240111).

\section{REFERENCES}

(1) Furukawa, S.; De Feyter, S. Templates in Chemistry III; Springer: Berlin/Heidelberg, 2009; Vol. 287, pp 87-133.

(2) Quan, Z.; Fang, J. Nano Today 2010, 5, 390-411.

(3) Kosterlitz, J. M.; Thouless, D. J. J. Phys. C: Solid State Phys. 1973, 6, 1181 .

(4) Nelson, D. R.; Halperin, B. I. Phys. Rev. B 1979, 19, 2457-2484.

(5) Young, A. P. Phys. Rev. B 1979, 19, 1855-1866.

(6) Bernard, E. P.; Krauth, W. Phys. Rev. Lett. 2011, 107, 155704.

(7) Frenkel, D.; Eppenga, R. Phys. Rev. A 1985, 31, 1776-1787.

(8) Cuesta, J. A.; Frenkel, D. Phys. Rev. A 1990, 42, 2126-2136.

(9) Bates, M. A.; Frenkel, D. J. Chem. Phys. 2000, 112, 10034-10041.

(10) Lagomarsino, M. C.; Dogterom, M.; Dijkstra, M. J. Chem. Phys. 2003, 119, 3535-3540.

(11) Wojciechowski, K. W.; Frenkel, D. Comp. Met. Sci. Technol. 2004, 10, 235-255. 
(12) Donev, A.; Burton, J.; Stillinger, F. H.; Torquato, S. Phys. Rev. B 2006, 73, 054109.

(13) Zhao, K.; Bruinsma, R.; Mason, T. G. Proc. Natl. Acad. Sci. U.S.A.

2011, 108, 2684-2687.

(14) Avendano, C.; Escobedo, F. A. Soft Matter 2012, 8, 4675-4681.

(15) Manna, L.; Scher, E. C.; Alivisatos, A. P. J. Am. Chem. Soc. 2000, $122,12700-12706$.

(16) Sun, Y.; Xia, Y. Science 2002, 298, 2176-2179.

(17) Zhao, N.; Qi, L. Adv. Mater. 2006, 18, 359-362.

(18) Quilliet, C.; Zoldesi, C.; Riera, C.; van Blaaderen, A.; Imhof, A. Eur. Phys. J. E 2008, 27, 13-20.

(19) Deka, S.; Miszta, K.; Dorfs, D.; Genovese, A.; Bertoni, G.; Manna, L. Nano Lett. 2010, 10, 3770-3776.

(20) Miszta, K.; de Graaf, J.; Bertoni, G.; Dorfs, D.; Brescia, R.; Marras, S.; Ceseracciu, L.; Cingolani, R.; van Roij, R.; Dijkstra, M.; Manna, L. Nat. Mater. 2011, 10, 872-876.

(21) Henzie, J.; Grünwald, M.; Widmer-Cooper, A.; Geissler, P. L.; Yang, P. Nat. Mater. 2012, 11, 131-7.

(22) Yin, J. S.; Wang, Z. L. Phys. Rev. Lett. 1997, 79, 2570-2573.

(23) Maeda, H.; Maeda, Y. Phys. Rev. Lett. 2003, 90, 018303.

(24) Haji-Akbari, A.; Engel, M.; Keys, A. S.; Zheng, X.; Petschek, R. G.; Palffy-Muhoray, P.; Glotzer, S. C. Nature 2009, 462, 773.

(25) Torquato, S.; Jiao, Y. Nature 2009, 460, 876.

(26) Marechal, M.; Kortschot, R. J.; Demirörs, A. F.; Imhof, A.; Dijkstra, M. Nano Lett. 2010, 10, 1907-1911.

(27) Marechal, M.; Dijkstra, M. Phys. Rev. E 2010, 82, 031405.

(28) de Graaf, J.; van Roij, R.; Dijkstra, M. Phys. Rev. Lett. 2011, 107, 155501.

(29) Nguyen, T. D.; Jankowski, E.; Glotzer, S. C. ACS Nano 2011, 5, 8892-8903.

(30) Blaak, R.; Mulder, B. M. Phys. Rev. E 1998, 58, 5873-5884.

(31) Sinkovits, D. W.; Luijten, E. Nano Lett. 2012, 12, 1743-1748.

(32) Glotzer, S. C. Nature 2012, 481, 450-452.

(33) Deegan, R. D.; Bakajin, O.; Dupont, T. F.; Huber, G.; Nagel, S. R.; Witten, T. A. Nature 1997, 389, 827-829.

(34) Filion, L.; Marechal, M.; van Oorschot, B.; Pelt, D.; Smallenburg, F.; Dijkstra, M. Phys. Rev. Lett. 2009, 103, 188302.

(35) Qi, W.; de Graaf, J.; Qiao, F.; Marras, S.; Manna, L.; Dijkstra, M. To be submitted for publication. 